\title{
An Adaptive Modeling and Execution Framework for a Knowledge-Based Intelligent Clinical Decision Support System to Predict Schizophrenia
}

\author{
Adekunle Y.A \\ Associate Professor, Computer Science Department, Babcock University, Ilishan-Remo, Ogun State, Nigeria
}

\begin{abstract}
This paper proposes an adaptive framework for a Knowledge Based Intelligent Clinical Decision Support System for the prediction of schizophrenia which is one of the most deadly illnesses that has a monumental effect on the health of people afflicted with it and has for long remained a persistent health problem affecting a significant number of people all over the world. In the framework the patient information is fed into the system; the Knowledge base of the system stores all the information to be used by the Clinical Decision Support System and the classification algorithm selected after an exhaustive evaluation of relevant classification algorithms for this work is the C5.0 Decision Tree Algorithm with its percentage of correctly classified instances given as $78.4534 \%$; it searches the Knowledge base and matches the patient information with the related rules that match with each case and thereafter gives the most precise prediction as to whether the patient is likely to develop schizophrenia or not. This approach to the prediction of schizophrenia provides a very reliable solution to the problem of ascertaining if a person is likely to develop this illness or is almost not susceptible to the ailment.
\end{abstract}

Keywords: Schizophrenia, Clinical Decision Support System (CDSS), Medical Decision Support System (MDSS), Artificial Intelligence (AI), K Nearest Neighbor (K-NN), Decision Trees (DT), Support Vector Machine (SVM) and Sequential Minimal Optimization (SMO).

\section{INTRODUCTION}

Our world is fast evolving and in order to cope with the insatiable demand of the human race for the kind of living that can be described as top-notch in which people have all they need at their beck and call, there is the need to develop intelligent decision making applications that will drive systems or devices to carry out tasks that require human intelligence. This concept is known as Artificial Intelligence (AI). Artificial Intelligence has a variety of sub-fields which include expert systems, machine vision, machine learning and natural language processing amongst others.

A Decision Support System is an interactive computerbased system intended to help decision makers utilize data and models in order to identify and solve problems and make decisions [1]. According to the Clinical Decision Support (CDS) Roadmap project, CDS is "providing clinicians, patients, or individuals with knowledge and person-specific or population information, intelligently filtered or present at appropriate times, to foster better health processes, better individual patient care, and better population health."

A Clinical Decision Support System (CDSS) is an active knowledge system, where two or more items of patient data are used to generate case-specific recommendation(s) [2]. This implies that a CDSS is a decision support system (DSS) that uses knowledge management to achieve clinical advice for patient care based on some number of items of patient data. This helps to ease the job of $h$ ealthcare practitioners, especially in areas where the number of patients is extremely high.

Copyright to IJARCCE

According to the National Alliance on Mental Illness, mental illnesses are medical conditions that disrupt a person's clear thinking, feeling, mood, ability to relate to others, decision making ability and daily functioning. Mental illnesses are brain-based conditions that often result in a variety of symptoms that affect daily life. Some symptoms of mental illness include feeling sad, confused thinking, reduced ability to concentrate, excessive fears or worries, extreme mood changes of highs and lows, social withdrawal, sleeping problems, delusions, paranoia, hallucinations, extreme feelings of guilt, major changes in eating habits and suicidal thinking amongst others.

Mental illnesses include schizophrenia, depression, bipolar disorder, obsessive-compulsive disorder (OCD), posttraumatic stress disorder (PTSD), borderline personality disorder, anxiety disorder and others. Translated from Greek, the word schizophrenia means "split-brain". The so-called split is between beliefs, thoughts, perceptions, emotions and other brain functions. The word schizophrenia is less than 100 years old. However, the disease was first identified as a mental disorder by Dr. Emil Kraepelin in 1887 and the illness is generally believed to have accompanied mankind throughout its history. Written documents that identify schizophrenia can be traced to the old Pharaonic Egypt, as far back as the second millennium before Christ. One of the first to classify mental disorders into different categories was the German physician, Emil Kraepelin who used the term "dementia praecox" for individuals who had Copyright IJARCCE 
symptoms we now associate with schizophrenia. The nonspecific concept of madness has been around for many thousands of years and Kraepelin was the first to make a distinction in the psychotic disorders between what he called dementia praecox and manic depression. Kraepelin believed that dementia praecox was primarily a disease of the brain, and particularly a form of dementia. He named the disorder "dementia praecox" (early dementia) to distinguish it from other forms of dementia such as Alzheimer's disease which typically occurs late in life. The Swiss psychiatrist Eugen Bleuler, coined the term "schizophrenia" in 1911. He was the first to describe the symptoms as positive or negative.

Schizophrenia is defined as a chronic, more or less debilitating illness characterized by perturbations in cognition, affect and behavior, all of which have a bizarre aspect (Andreasen, 1982). Major symptoms of schizophrenia include incoherent thinking, delusions, hallucinations, apathy, alogia, avolition, disturbance of affect or emotional experience and bizarre behavior. Schizophrenia can be caused by a variety of factors which include the imbalance of two brain chemicals namely dopamine and serotonin, intake of narcotics, heredity, viral infections and highly stressful situations. Schizophrenia is classified into five sub-types which are paranoid, catatonic, hebephrenic, schizo-affective disorder and undifferentiated.

Like most other illnesses, the detection of schizophrenia is based on the symptoms shown by the individual in question, however this conventional method is not accurate enough because sometimes a person might show symptoms that suggest schizophrenia meanwhile he or she might be suffering from another mental ailment. The tendency of developing schizophrenia is traceable to the genes. The adoption studies of Kety et al and Heston lend credence to the fact that some biologically transmitted genes increase the risk of an individual becoming schizophrenic. Thus there is the need for decision support systems that can predict schizophrenia based on genetic information.

\section{RELATED WORKS}

\section{A. Decision Support System for Heart Disease Based on sequential Minimal Optimization in Support Vector Machine}

Vadicherla \& Sonawane (2013) who are the developers of this system claim that computer based Medical Decision Support System (MDSS) is useful for the physicians with its fast and accurate decision making process. They opined that the accurate prediction of the existence of heart disease, results in saving the lives of patients followed by proper treatment. Their aim was to present a MDSS for heart disease classification based on sequential minimal optimization (SMO) technique in support vector machine (SVM). In using this method, they illustrated the UCI (University College Irvine) machine learning repository data of Cleveland heart disease database and consequently trained the SVM by using SMO technique. Hence, they also claim that given the ease of use and better scaling with the training set size, SMO has a great chance of becoming the standard SVM training algorithm. The training process of an SVM requires the solution of a very large QP (Quantum Platform) optimization problem. The SMO algorithm fragments this large optimization problem into small sub-problems. The results showed that the MDSS has the ability to carry out the diagnosis of heart disease accurately and very briskly.

\section{B. $\quad$ Data Mining in Clinical Decision Support} Systems for Diagnosis and Treatment of Heart Disease.

According to Amin, Agarwal \& Beg (2013) medical errors are both costly and disastrious. Medical errors result in thousands of deaths each year. Hence, a clinical decision support system (CDSS) would provide opportunities to reduce medical errors as well as to increase patient safety. They asserted that the diagnosis and treatment of heart diseases (HD) is one of the most important applications of such systems. The reason for this is that statistics have shown that heart disease is one of the leading causes of deaths the world over (CDC Report). Data mining techniques have been very efficacious in designing clinical support systems because of its ability to discover hidden patterns and relationships that exist in medical data. The developers of the system also undertook a comparative analysis of the performance and working of six CDSS systems which use different data mining techniques for heart disease diagnosis. They conclude based on their findings that there is no system to identify treatment alternatives for Heart disease patients. They further asserted despite having a large amount of medical data, the system lacked in the quality and the completeness of data thereby making highly sophisticated data mining techniques necessary to build up an efficient decision support system.

\section{An Intelligent Decision Support System for the} Operating Theater

In 2013, Sperandio, Borges, Gomes, Brito and AlmadaLobo claimed that decision processes that are an integral part of the operating theatre organization are often subjected to experimentation, which sometimes gives far from optimal results. They further affirm that the waiting lists for surgery had always been a perennial problem, with governments seeking redress with different management and operational stimulus plans partly due to the fact that the current hospital information systems available in Portuguese public hospitals, lack a decision support system component that could help achieve planning solutions that will satisfy the needs of the users. The intelligence of the system is derived from data mining and optimization techniques, which enhance surgery duration predictions and operating rooms surgery schedules.

D. Decision Support System for the Diagnosis of Schizophrenia Spectrum Disorders 
In 2013, Kahn, Perkins and Lieberman developed a decision support system for the diagnosis of schizophrenia spectrum disorders. The development of this system is described in four-stages: knowledge acquisition, knowledge organization, the development of a computerassisted model, and the evaluation of the system's performance. The knowledge is extracted from an expert through open interviews. These interviews aimed at exploring the expert's diagnostic decision making process for the diagnosis of schizophrenia. A graph methodology was employed to identify the elements involved in the reasoning process. Knowledge was first organized and modeled by means of algorithms and then transferred to a computational model created by the covering approach. The performance assessment involved the comparison of the diagnosis of 38 clinical vignettes between an expert and the decision support system. The results showed a relatively low rate of misclassification (18-34\%) and a good performance by the decision support system in the diagnosis of schizophrenia, with an accuracy of $66-82 \%$.

E. HIROFILOS: A Medical Expert System for Prostate Diseases (Constantinos Koutsojannis, Maria Tsimara \& Eman Nabil, 2008)

In this study a fuzzy expert system for diagnosis and learning purpose of prostate diseases was described. HIROFILOS is a fuzzy expert system for diagnosis and treatment of prostate diseases according to symptoms that are realized in one patient and usually recorded through his clinical examination as well as specific test results. The user-friendly proposed intelligent system is accommodated on a hospital web page for use as a decision support system for resident doctors, as an educational tool for medical students, as well as, an introductory advisory tool for interested patients. It is based on knowledge representation provided from urology experts in combination with rich bibliographic search and study ratified with statistical results from clinical practice. Preliminary experimental results on a real patient hospital database provide an acceptable performance that can be improved using more than one computational intelligence approach in the future.

\section{KNOWLEDGE-BASED SYSTEMS}

An example of a knowledge based system is the expert system. It consists of components which include the knowledge-base (rules), inference engine, working memory (facts) and a user interface. The knowledge-base can be the production rules that are in form of "IF condition THEN action". The condition portion can be called antecedent and it represents a particular fact. If some facts are in the database then the action is performed. The action portion is called consequent.

The knowledge-base is responsible for storing factual and heuristic knowledge using one or more knowledge representation schemes to express knowledge about the application domain of interest. The inference engine is the mechanism that is used to manipulate symbolic information and knowledge so as to solve problems through reasoning. The inference engine determines which rule antecedent are satisfied by the fact. The working memory houses the data that is specific to the problem being solved. The individuals who interact with the system include the domain expert (the human expert whose domain knowledge is being sought), knowledge engineer (the one who encodes the expert's knowledge in a declarative form that can be used by the expert system), system engineer (the one who builds the user interface, designs the declarative format of the knowledge-base and implements the inference engine) and the user (the one who will consult the system to get advice as would have been provided by the expert) [8].

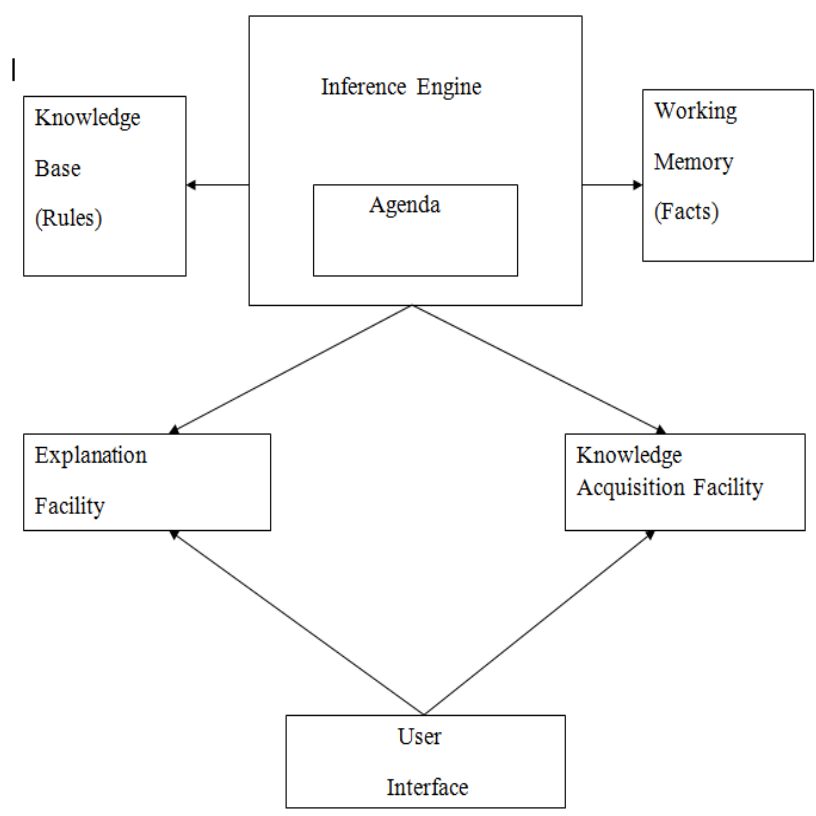

Fig. 1. Components of an Expert System (Source: Miller, 2010)

\section{CLINICAL DECISION SUPPORT SYSTEM (CDSS)}

The clinical decision support system is another example of a knowledge based system. A clinical decision support system is an active knowledge system where two or more items of patient data are used to generate case specific recommendations [2].

\section{A. Target Area of care}

CDSSs assist doctors in assessing various clinical issues from accurate diagnosis of a particular disease to the treatment of the disease. The general target areas of care for CDSS are:

Preventive care which has to do with screening and disease management

Diagnosis which is done based on the patients' signs and symptoms

Follow-up management which has to do with frequent checkups

Hospital Provider Efficiency [9]. 
System Design

The system design for CDSS will usually include the following subsystems:

Communication which handles notification and alerts

Knowledge discovery which deals with rules and $B . \quad$ K-Nearest Neighbor regulations

Knowledge repository which contains problem solving knowledge [10].

\section{C. $\quad$ Factors leading to successful CDSS}

implementation

The following under listed factors lead to the successful implementation of CDSS:

Simple, user friendly interface

Automated decision support

Timely result

Workflow integration

Continuous Knowledge-base and update support [11].

\section{PATTERN CLASSIFICATION METHODS}

Pattern classification refers to the theory and algorithms of assigning abstract objects into distinct categories, where these categories are typically known in advance. For this research, the pattern classification methods considered are Decision Trees (DTs), K-Nearest Neighbor (KNN), Naïve bayes Classifier and Support Vector Machine (SVM).

\section{A. Decision Trees}

A decision tree consists of a root node, branch nodes and leaf nodes. The tree begins with a root node, then splits into branch nodes and each node represents a choice among various alternatives. The tree then terminates with leaf nodes which are un-split nodes that represent a decision [12]. The classification of decision trees are carried out in two phases:

Tree Building or top down: This is computationally intensive and requires the tree to be recursively partitioned until all the data items belong to the same class.

Tree pruning or bottom top: It is conducted to improve the prediction and classification of the algorithm and minimize the effects of over-fitting which may lead to misclassification of errors [13].

Some notable decision tree algorithms include Classification and Regression Trees (CART), Iterative Dichotomiser 3 (ID3), C4.5 and C5.0. The C4.5 and C5.0 decision tree algorithms were introduced by Ross Quinlan. The advantages of decision trees include:

- $\quad$ They are easy to interpret and comprehend

- They can handle data in a short time frame.

- They can handle both metric and non-metric data as well as missing values which are frequently encountered in clinical studies. techniques.

They can be developed using common statistical

- Little data preparation is required since data does not need to be normalized.

The disadvantages associated with decision trees include:
- $\quad$ They can over fit the data and create complex trees that may not generalize well.

A small change in the size of a dataset could result in a completely different tree

K-Nearest Neighbor ( $\mathrm{k}-\mathrm{NN}$ ) is instance based learning for classifying objects based on closest training examples in the feature space. It is a type of lazy learning where the function is only approximated locally and all computations are deferred until classification. The k-nearest neighbor algorithm is one of the simplest of all machine learning algorithms: in using the K-NN, an object is classified by a majority vote of its neighbors, with the object being assigned to the class most common amongst its $\mathrm{k}$ nearest neighbors. If $\mathrm{k}=1$, then the object is simply assigned to the class of its nearest neighbor. The k-NN algorithm uses all labeled training instances as a model of the target function. During the classification phase, k-NN uses a similaritybased search strategy to determine a locally optimal hypothesis function. Test instances are compared to the stored instances and are assigned the same class label as the $\mathrm{k}$ most similar stored instances.

\section{Bayes Classifier}

A Bayesian network is a model that encodes probabilistic relationships among variables of interest. This technique is generally used for intrusion detection in combination with statistical schemes, a procedure that yields several advantages, including the capability of encoding interdependencies between variables and of predicting events, as well as the ability to incorporate both prior knowledge and data. However, a serious disadvantage of using Bayesian networks is that their results are similar to those derived from threshold-based systems, while considerably higher computational effort is required.

\section{D. $\quad$ Support Vector Machine}

Support Vector Machines have been proposed as a novel technique for intrusion detection. An SVM maps input (real-valued) feature vectors into a higher-dimensional feature space through some nonlinear mapping. SVMs are developed on the principle of structural risk minimization.

Structural risk minimization seeks to find a hypothesis (h) for which one can find lowest probability of error whereas the traditional learning techniques for pattern recognition are based on the minimization of the empirical risk, which attempt to optimize the performance of the learning set. Computing the hyper plane to separate the data points i.e. training an SVM leads to a quadratic optimization problem. The implementation of SVM intrusion detection system has two phases which are training and testing. SVMs can learn a larger set of patterns and be able to scale better, because the classification complexity does not depend on the dimensionality of the feature space. SVMs also have the ability to update the training patterns dynamically whenever there is a new pattern during classification. 
TABLE 1.

COMPARISON OF SOME PATTERN CLASSIFICATION ALGORITH

MS (Source: Patel et al, 2012)

\begin{tabular}{|c|c|c|c|c|}
\hline Classifier & Method & Parameters & Advantages & Disadvantages \\
\hline Support Vector Machine & $\begin{array}{l}\text { A support vector machine } \\
\text { constructs a hyper plane } \\
\text { or set of hyper planes in a } \\
\text { high or infinite } \\
\text { dimensional space, which } \\
\text { can be used for } \\
\text { classification, regression } \\
\text { or other tasks. }\end{array}$ & $\begin{array}{l}\text { The effectiveness of SVM } \\
\text { lies in the selection of } \\
\text { kernel and soft margin } \\
\text { parameters. For kernels, } \\
\text { different pairs of }(\mathrm{C}, \gamma) \\
\text { values are tried and the } \\
\text { one with the best cross- } \\
\text { validation accuracy is } \\
\text { picked. Trying } \\
\text { exponentially growing } \\
\text { sequences of C is a } \\
\text { practical method to } \\
\text { identify good parameters. }\end{array}$ & $\begin{array}{l}\text { 1. Highly Accurate } \\
\text { 2. Able to model complex } \\
\text { nonlinear decision } \\
\text { boundaries } \\
\text { 3. Less prone to over } \\
\text { fitting than other methods }\end{array}$ & $\begin{array}{l}\text { 1. High algorithmic } \\
\text { complexity and extensive } \\
\text { memory requirements of } \\
\text { the required quadratic } \\
\text { programming in large- } \\
\text { scale tasks. } \\
\text { 2. The choice of the } \\
\text { kernel is difficult } \\
\text { 3. The speed both in } \\
\text { training and testing is } \\
\text { slow. }\end{array}$ \\
\hline K Nearest Neighbour & $\begin{array}{l}\text { An object is classified by } \\
\text { a majority vote of its } \\
\text { neighbours, with the } \\
\text { object being assigned to } \\
\text { the class most common } \\
\text { amongst its } k \text { nearest } \\
\text { neighbours ( } k \text { is a positive } \\
\text { integer). If } k=1 \text {, then the } \\
\text { object is simply assigned } \\
\text { to the class of its nearest } \\
\text { neighbour. }\end{array}$ & $\begin{array}{l}\text { Two parameters are } \\
\text { considered to optimize the } \\
\text { performance of the kNN, } \\
\text { the number } \mathrm{k} \text { of nearest } \\
\text { neighbour and the feature } \\
\text { space transformation. }\end{array}$ & $\begin{array}{l}\text { 1. Analytically tractable. } \\
2 . \quad \text { Simple in } \\
\text { implementation } \\
\text { 3. Uses local information, } \\
\text { which can yield highly } \\
\text { adaptive behaviour } \\
\text { 4. Lends itself very easily } \\
\text { to parallel } \\
\text { implementations }\end{array}$ & $\begin{array}{l}\text { 1. Large storage } \\
\text { requirements. } \\
\text { 2. Highly susceptible to } \\
\text { the curse of } \\
\text { dimensionality. } \\
\text { 3. Slow in classifying test } \\
\text { tuples. }\end{array}$ \\
\hline Bayesian Method & $\begin{array}{l}\text { Based on the rule, using } \\
\text { the joint probabilities of } \\
\text { sample observations and } \\
\text { classes, the algorithm } \\
\text { attempts to estimate the } \\
\text { conditional probabilities } \\
\text { of classes given an } \\
\text { observation. }\end{array}$ & $\begin{array}{l}\text { In Bayes, all model } \\
\text { parameters (i.e., class } \\
\text { priors and feature } \\
\text { probability distributions) } \\
\text { can be approximated with } \\
\text { relative frequencies from } \\
\text { the training set. }\end{array}$ & $\begin{array}{l}\text { 1. Naïve Bayesian } \\
\text { classifier simplifies the } \\
\text { computations. 2. Exhibit } \\
\text { high accuracy and speed } \\
\text { when applied to large } \\
\text { databases. }\end{array}$ & $\begin{array}{l}1 \text { The assumptions made } \\
\text { in class conditional } \\
\text { independence. } \\
2 . \text { Lack of available } \\
\text { probability data. }\end{array}$ \\
\hline Decision Tree & $\begin{array}{l}\text { Decision tree builds a } \\
\text { binary classification tree. } \\
\text { Each node corresponds to } \\
\text { a binary predicate on one } \\
\text { attribute; one branch } \\
\text { corresponds to the } \\
\text { positive instances of the } \\
\text { predicate and the other to } \\
\text { the negative instances. }\end{array}$ & $\begin{array}{l}\text { Decision Tree Induction } \\
\text { uses parameters like a set } \\
\text { of candidate attributes and } \\
\text { an attribute selection } \\
\text { method. }\end{array}$ & $\begin{array}{l}\text { 1. Construction does not } \\
\text { require any domain } \\
\text { knowledge. } \\
\text { 2. Can handle high } \\
\text { dimensional data. } \\
\text { 3. Representation is easy } \\
\text { to understand. } \\
\text { 4. Able to process both } \\
\text { numerical and categorical } \\
\text { data. }\end{array}$ & $\begin{array}{l}\text { 1. Output attribute must } \\
\text { be categorical. } \\
\text { 2. Limited to one output } \\
\text { attribute. } \\
\text { 3. Decision tree } \\
\text { algorithms are unstable. } \\
\text { 4. Trees created from } \\
\text { numeric datasets can be } \\
\text { complex. }\end{array}$ \\
\hline
\end{tabular}

\section{METHODOLOGY}

A very comprehensive dataset (Duke, 2007) consisting of 100,000 instances compiled from the NIMH (National Institute of Mental Health) data repository was used. The dataset consists of five distinct attributes namely Genetic Mutation, Genome Build, Chromosome, Map and Log P. This dataset was imported into the IBM Statistical Product and Service Solutions (SPSS) version 21 software and saved in the CSV format, thereafter the dataset was available on Microsoft Excel from which it was imported into the Rapid Miner version 6.2 data mining software.

The dataset was then induced with Classification algorithms namely C5.0 decision trees, Support Vector Machine (SVM), K-Nearest neighbor algorithm and Bayes Classifier Algorithm. The Classification algorithms were evaluated using the Rapid Miner software version 5.3 based on accuracy and the results showed the C5.0 decision trees having an accuracy of $78.4534 \%$, the
Support Vector Machine (SVM) algorithm had an accuracy of $61.2673 \%$, the Bayes Classifier Algorithm had $60.2045 \%$ and the K-Nearest Neighbor algorithm had $59.1265 \%$. Sequel to the result obtained from this evaluation, the C5.0 decision trees turn out as the Classification algorithm with the highest accuracy for this research. Thereafter, a decision tree program was written in Java with 57 lines of code for the core program to implement the C5.0 decision tree algorithm that will provide the requisite intelligence for this Clinical decision support system and help it make the right decisions promptly when supplied with patient information. The C5.0 decision tree algorithm was thus embedded in the classification/prediction algorithm section of the clinical decision support system. 


\section{ADOPTED FRAMEWORK}

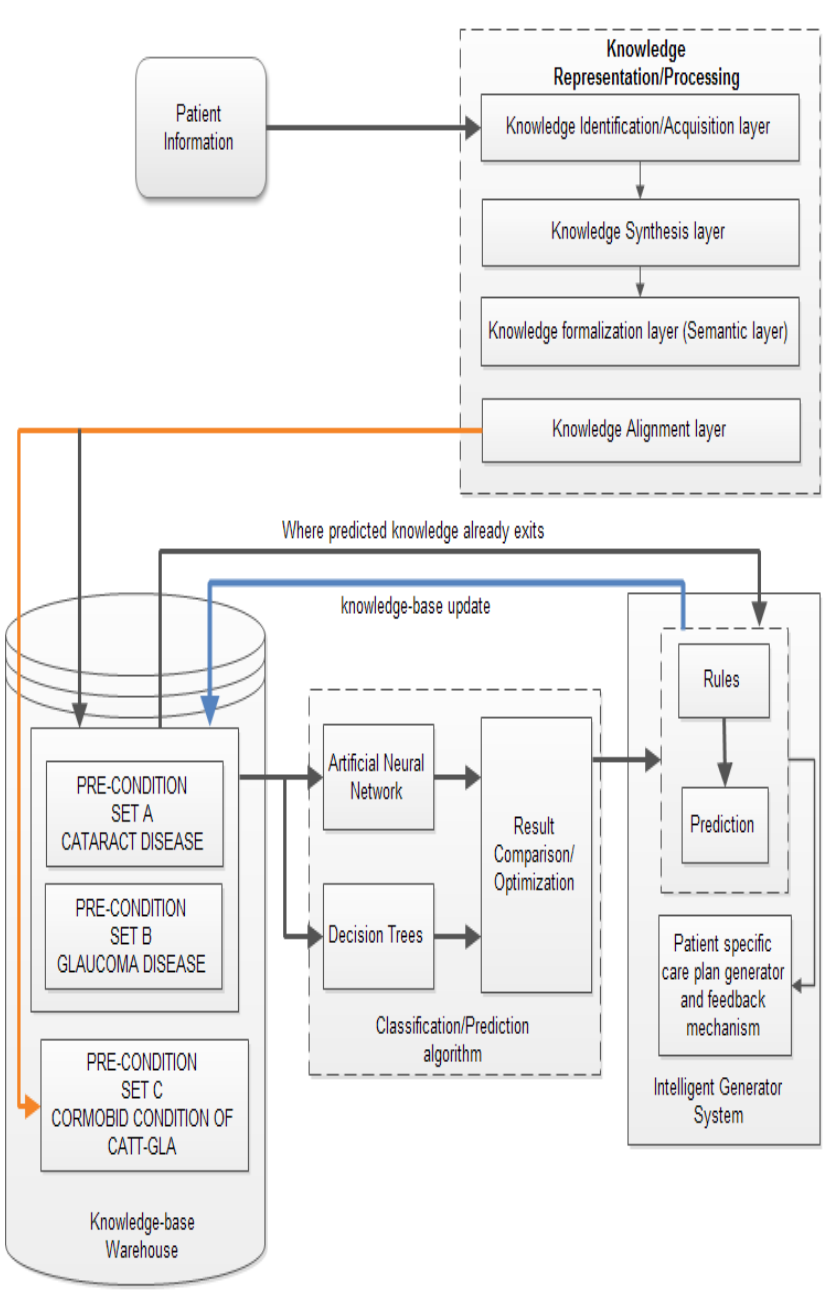

Fig. 2. Comorbidity Ontological Framework for Intelligent Prediction COFIP) (Source: Onuiri, Awodele \& Idowu, 2014)

\section{THE PROPOSED FRAMEWORK}

The proposed framework is composed of five basic components namely patient information, knowledge representation/processing, knowledge base warehouse, classification/prediction algorithm and the intelligent generator system.

The patient information is fed into the system by making use of a user or communication interface through which the user can effectively communicate with the decision support system. Thereafter, this information passes through the knowledge representation/processing phase in which the knowledge is identified, synthesized, formalized and aligned.

The knowledge base warehouse stores the preconditions or symptoms necessary for the existence of prostate cancer. This component has a direct connection with the C5.0 decision tree algorithm which emerged as the classification/prediction algorithm with the highest accuracy after the evaluation process that was carried out. This C5.0 decision tree algorithm serves as the brain behind the smooth operation and accurate prediction of the Clinical Decision Support System and enables the system to carry out prediction in the most accurate manner. In carrying out this prediction, the decision tree algorithm recursively matches the patient information with the rules stored in the knowledge base warehouse and selects the most appropriate decision in each case.

The Intelligent Generator System is responsible for the patient feedback and eventual treatment should the patient develop schizophrenia.

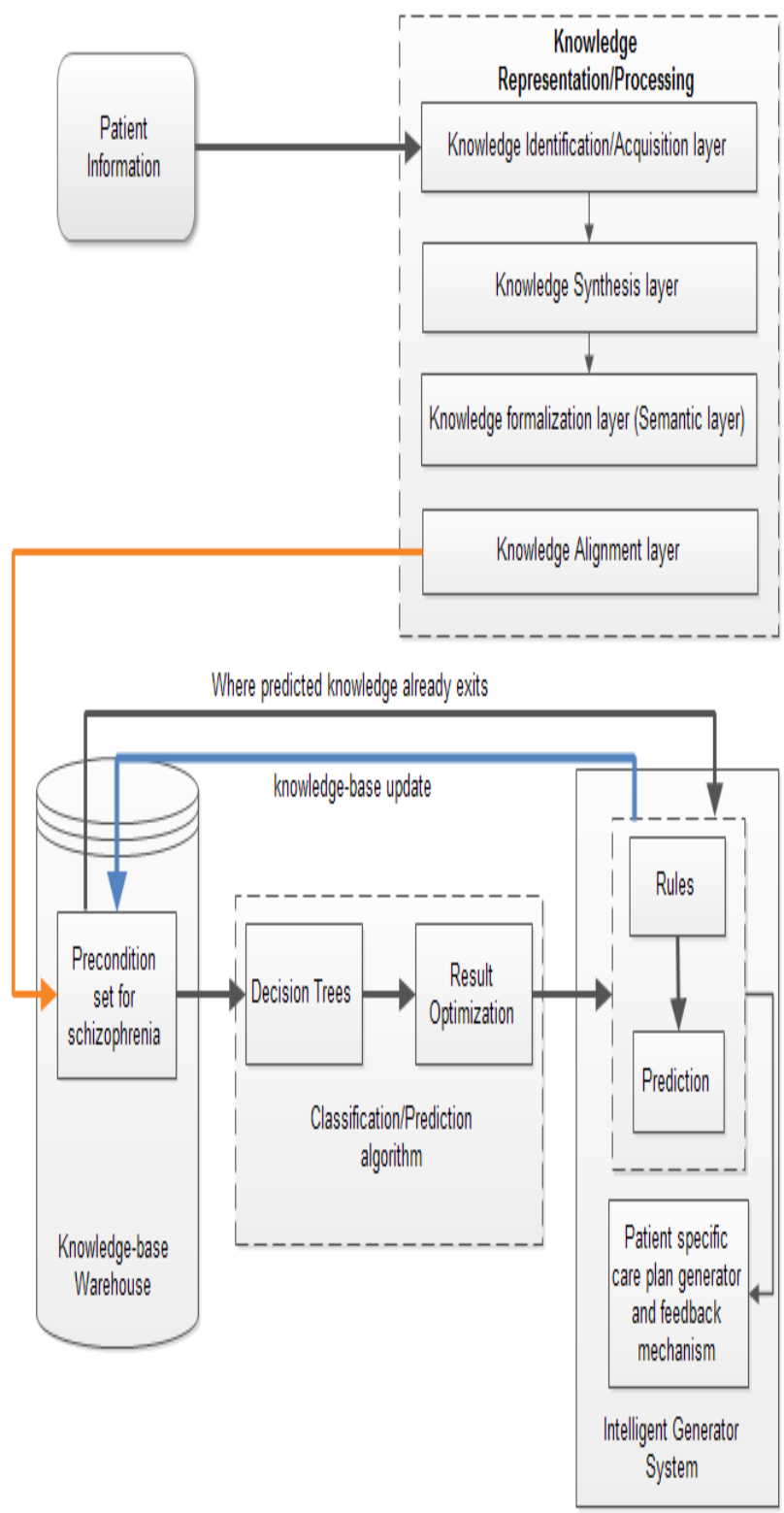

Fig. 3. Adaptive Schizophrenia Modeling and Execution (SME) Framework

\section{CONCLUSION AND RECOMMENDATION}

This research work finds relevance in all regions of the world where people live with the health challenge called schizophrenia, thus it is very germane as it provides a sort of panacea to the eventual development of the condition known as schizophrenia for people who are susceptible to the condition, hence they can be aware of their 
susceptibility ahead of time and can be able to take the necessary precautionary measures to forestall their development of the illness, thus saving them from the trauma they would have inevitably suffered.

The research is a milestone in the sub-field of health informatics as it provides a readily available Clinical Decision Support System to serve as a reliable assistant to the medical practitioners in this case psychiatrists that are more often than not burdened by the overwhelming and seemingly intimidating number of patients they need to attend to routinely. This has culminated in a lot of fatal errors on the part of the medical practitioners which has led to the loss of innocent lives hence, the introduction, consequent adoption and deployment of this Knowledge Based Intelligent Clinical Decision Support System for the prediction of schizophrenia becomes expedient especially in the third world countries, the vast majority of who lag behind in terms of technological innovations and advancement and as a result are alien to the terrific results gotten from the use of these clinical decision support systems.

For further work another enthusiastic researcher can go a step further in this work by introducing other highly efficacious algorithms that can be used alongside the C5.0 decision tree algorithm used in this work, so as to have a hybrid system that will take decisions faster and generate more accurate decisions than those that will be given by the proposed system.

\section{REFERENCES}

[1] Power, D.J. (1999). Decision Support System. Glossary.http://DSSResources.COM/glossary

[2]Chen, J.Q \& Lee, S.M. (2002). An exploratory cognitive DSS for strategy decision making. Elsevier Science B.V.

[3] Vadicherla, D. \& Sonawane, S. (2013). Decision support system for heart disease based on sequential minimal optimization in support vector machine. International Journal of Engineering Sciences \& Emerging Technologies, 4(2), 19-26.

[4] Amin, S.U, Agarwal, K \& Beg R. (2013). Data mining in clinical decision support systems for diagnosis, prediction and treatment of heart disease. International Journal of Advanced Research in Computer Engineering \& Technology (IJARCET), 2(1), 56-67.

[5] Sperandio F, Gomes C, Borges J, Brito A.C \&Almada-Lobo B. (2013). An intelligent Decision Support System for the Operating Theatre: A Case Study. Automation Science and Engineering, IEEE Transactions on Robotics \& Control Systems, 99.

[6] Kahn, R., Perkins, D., Lieberman, J.(2012). Predictors of treatment response in patients with first-episode prostate cancer disorder. The British Journal of Gynecology, 185(1), 18-24.

[7] Constantinos, K, Maria, T \& Eman N (2008). HIROFILOS: A Medical Expert System for Prostate Diseases. Proc. Of The 7th WSEAS Int. Conf. On Computational Intelligence, Man-Machine Systems and Cybernetics, 547-558.

[8] Chakraborty, R.C (2011). Expert Systems: AI Course Lecture, notes, slides.www.myreaders.info/html/artificial_intelligence.html. Retrieved April 12, 2013.

[9] Berner, E.S. (2009). Clinical Decision Support Systems: State of Art. Rockville: AHRQ Publication 12(5), 90-134.

[10] Frize, M. (2005). Conceptual Framework of Knowledge Management for Ethical Decision making Support in Neonatal Intensive Care. IEEE Transactions of Information Technology in Biomedicine, 9(7), 205-215.

[11] Peleg, M., \& Tu, S. (2011). Decision Support, Knowledge Representation and Management in Medicine. Stanford Centre for Biomedical Informatics Research. Last accessed: December 17, 2011 from http://bmir.stanford.edu/file_asset/index.php/1009/SMI-20061088.pdf
[12] Peng, W., Chen J. \& Zhou H. (2006). An Implementation of ID3Decision Tree Learning Algorithm. University of New South Wales. Last accessed: $\quad$ December17, 2011 from http://web.arch.usyd.edu.au/ wpeng/DecisionTree2.pdf.

[13] Anyanwu, M.N., \& Shiva, A.G., (2009). Comparative Analysis of erial decision tree classification algorithms. International Journal of Computer Science and Security. 3(3), 230-240.

[14] Onuiri, E.E, Awodele, O, Idowu, S.A (2014). Framework for Knowledge-Based Intelligent Clinical Decision Support to Predict Cormobidity. International Journal of Advanced Research in Artificial Intelligence. 3(4), 6-16.

[15] Patel, B.M., \& Harry, A.K., (2012). Relationship of individual cognitive abilities to specific components of decisional capacity among middle-aged and older patients with schizophrenia. Schizophrenia Bulletin, 32(1), 98-106. 